\title{
Detecting variable (V), diversity (D) and joining (J) gene segment recombination using a two-colour fluorescence system
}

\author{
Gina B Scott, Erika A de Wynter, Graham P Cook
}

\begin{abstract}
Background: Diversity of immunoglobulins and the $T$ cell antigen receptors is achieved via the recombination activating gene (RAG)-mediated rearrangement of variable $(V)$, diversity $(D)$ and joining $(J)$ gene segments, and this underpins the efficient recognition of a seemingly limitless array of antigens. Analysis of $V(D) J$ recombination activity is typically performed using extrachromosomal recombination substrates that are recovered from transfected cells and selected using bacterial transformation. We have developed a two-colour fluorescence-based system that simplifies detection of both deletion and inversion joining events mediated by RAG proteins.

Results: This system employs two fluorescent reporter genes that differentially mark unrearranged substrates and those that have undergone RAG-mediated deletion or inversion events. The recombination products bear the hallmarks of true $V(D) J$ recombination and activity can be detected using fluorescence microscopy or flow cytometry. Recombination events can be detected without the need for cytotoxic selection of recombination products and the system allows analysis of recombination activity using substrates integrated into the genome.

Conclusions: This system will be useful in the analysis and exploitation of the V(D)J recombination machinery and suggests that similar approaches could be used to replace expression of one gene with another during lymphocyte development.
\end{abstract}

\section{Background}

The antigen receptor loci of $\mathrm{B}$ and $\mathrm{T}$ lymphocytes exhibit a unique mechanism of control amongst the genes of multicellular organisms. The production of functional immunoglobulin (Ig) and T cell receptor (TCR) genes is accomplished through a tightly regulated process of recombination. Variable (V), diversity (D) and joining (J) gene segments of antigen receptor loci are assembled into a functional coding unit by a series of site-specific recombination events mediated by the products of recombination activating gene (RAG)1 and RAG2 [1]. Recombination is targeted to specific sites by the recombination signal sequences (RSS), which flank the gene segments. RSS motifs consist of a conserved heptamer (CACAGTG) separated from a conserved nonamer (ACAAAAACC) by a spacer of variable sequence of either 12 or 23 base pairs (bp). Recombination occurs

\footnotetext{
* Correspondence: g.p.cook@leeds.ac.uk

Leeds Institute of Molecular Medicine, University of Leeds, Leeds, UK
}

between an RSS with a 12-bp spacer (RSS12) and an RSS with a 23-bp spacer (RSS23) and the intervening DNA is either deleted or inverted depending upon the orientation of the two signals (Figure 1). Double strand breaks introduced at the RSS motifs by the RAG proteins are then resolved by non-homologous end joining. Two products are generated, a signal joint in which the RSS motifs are joined and a coding joint (Figure 1) in which the gene segments are joined [2].

Assays of $\mathrm{V}(\mathrm{D}) \mathrm{J}$ recombination have relied extensively upon the transfection of extrachromosomal plasmid substrates into RAG-expressing cell lines and the recovery of these plasmids in Escherichia coli [3-6]. Many of these substrates are designed such that $\mathrm{V}(\mathrm{D}) \mathrm{J}$ recombination allows expression of a selectable marker in bacteria [3-5]. This approach has been extremely valuable in dissecting the basic mechanisms of recombination. However, since these substrates are extrachromosomal, this approach cannot be used to analyse the effect of chromatin structure on the recombination process. Alternatives have 

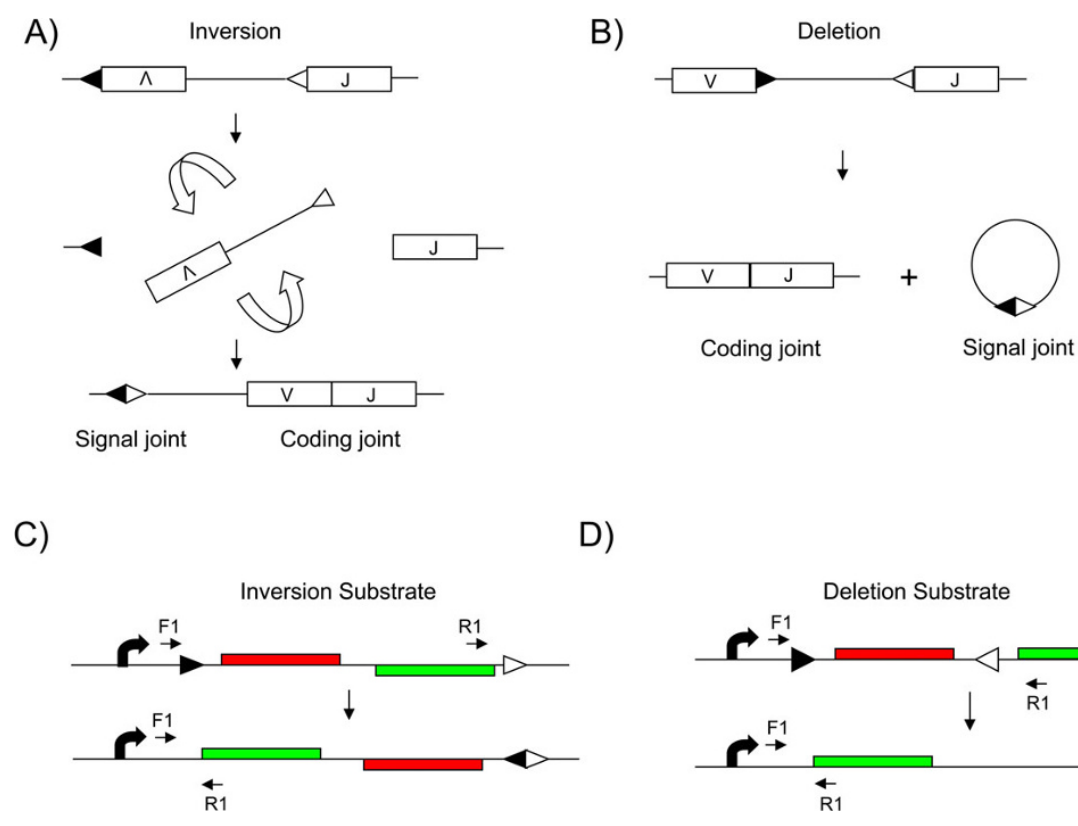

D)

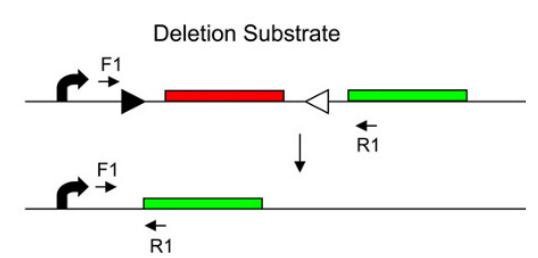

Figure 1 Physiological variable (V), diversity (D) and joining $(\mathrm{J})$ recombination and analogous recombination substrates. (a) $V$ and $J$ segments on opposite strands (as found in the human lg $\kappa$ locus) are joined by inversion between the recombination signal sequence (RSS) 12 (filled triangle) and RSS23 (open triangle) motifs to generate a linked signal joint and coding joint (the VJ rearrangement). (b) V and J segments located on the same strand (as found in the human $\lg \kappa$ and $\lambda$ loci) are recombined by deletion of the intervening DNA, leaving the coding segment on the chromosome and the signal joint on an excised circle of DNA. (c) In the inversion substrate, the DsRed gene and the EGFP gene are located on opposite stands, flanked by RSS12 and RSS23 motifs. V(D)J recombinase activity flips the segment allowing DsRed to be replaced by EGFP. (d) In the deletion substrate, the RSS motifs are in opposite orientations and flank the DsRed gene. On recombination, the DsRed gene is deleted, placing EGFP adjacent to the promoter. A single promoter is present in both constructs (curved arrow). The positions of the primer sequences F1 and R1, which were used to analyse recombination at the DNA level and for RT-PCR analysis, are shown.

been described which rely on the recombination of integrated genes encoding selectable markers [7-9], or recombination to generate a single fluorescent gene product [10-12]. Furthermore, some of these techniques have been applied in studies of recombination in transgenic mice [10]. Here, we describe a system whereby $\mathrm{V}$ (D)J recombination substrates are stably integrated into the host cell genome and both non-recombined and recombined products can be detected by fluorescence.

\section{Results and Discussion}

$\mathrm{V}(\mathrm{D}) \mathrm{J}$ recombination can occur either by deletion of the DNA between RSS motifs or by inversion of the intervening segment (Figure 1a, b). We generated vectors to assess both types of recombination. The system utilises two fluorescent proteins; DsRed, derived from the coral Discosoma and enhanced green fluorescent protein (EGFP). The system was designed such that unrearranged substrates would express the DsRed gene, whereas substrates that had undergone RAG-mediated recombination would replace DsRed expression with EGFP expression. In one construct, the EGFP open reading frame was placed on the opposite strand to DsRed and the RSS motifs arranged such that the segment containing DsRed and EGFP would be inverted by RAG-mediated recombination (inversion substrate; Figure 1c). In the other construct, the two reporter genes were in the same orientation, with the DsRed gene flanked by RSS12 and RSS23 motifs, allowing deletion of the DsRed gene by RAG-mediated recombination (deletion substrate; Figure 1d).

The inversion and deletion constructs were stably transfected into two cell lines representing different developmental stages of the $B$ cell lineage. The cell line 300-19P is a pre-B cell line, expresses RAG1 and RAG2 and is known to catalyse $\mathrm{V}(\mathrm{D}) \mathrm{J}$ recombination, whereas WEHI231 is a B cell line in which RAG gene expression has been silenced and is therefore unable perform $V(D) J$ recombination. The expression of RAG1 and RAG2 in these two cell lines was confirmed using reverse transcription polymerase chain reaction (RT-PCR) (Figure 2a). Pools of stable transfectants were analysed for recombination products at the DNA level using PCR. The results shown in Figure $2 b$ indicated that recombination of both deletion and inversion constructs was restricted to the RAG-expressing 300-19P cells.

These transfectants were then analysed for expression of the DsRed and EGFP reporter genes at the mRNA 
level. RT-PCR analysis established that expression of the EGFP gene was restricted to the RAG-expressing cell line and that EGFP transcripts could be detected in cells transfected with both the inversion and deletion construct (Figure 2c). A larger RT-PCR product resulting from the transcription through the DsRed open reading frame and continuing into EGFP was detected in cells transfected with the deletion substrate (Figure 2c).

Our aim was to identify $\mathrm{V}(\mathrm{D}) \mathrm{J}$ recombination at the single cell level. We therefore analysed expression of the DsRed and EGFP molecules by fluorescence. Fluorescence microscopy of the stably transfected RAG negative WEHI231 and RAG positive 300-19P cells indicated that expression of EGFP was restricted to the 300-19P cells. Furthermore, EGFP expression was clearly detectable in both the deletion and inversion transfectants (Figure 3a). Expression was further analysed by flow cytometry (Figure 3b). Untransfected WEHI231 and 300-19P cells were assigned an arbitrary fluorescence level of 1 unit and compared to the fluorescence observed following stable transfection with the deletion and inversion constructs. Expression of EGFP was detectable by flow cytometry in both deletion and inversion transfectants with the number of EGFP-expressing cells greater in the deletion substrate transfected population than the inversion substrate population. The PCR-based detection of DNA

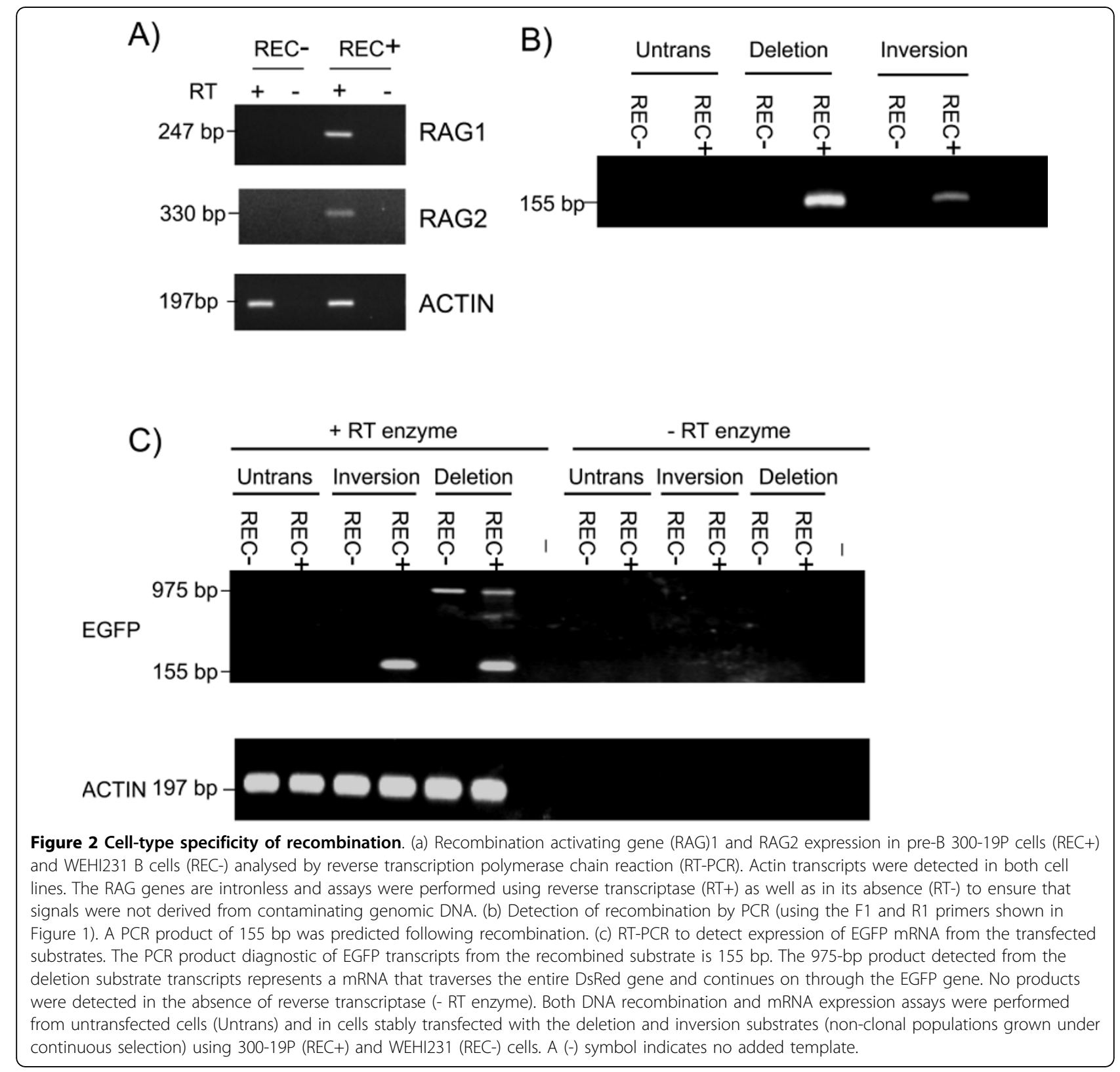




\section{Figure 3}

A)

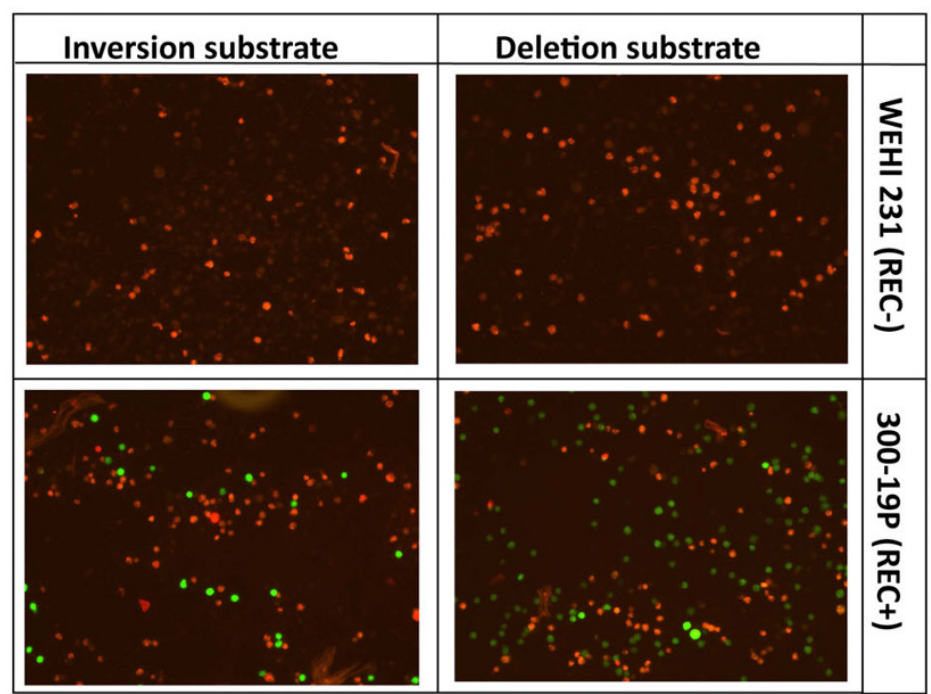

B)

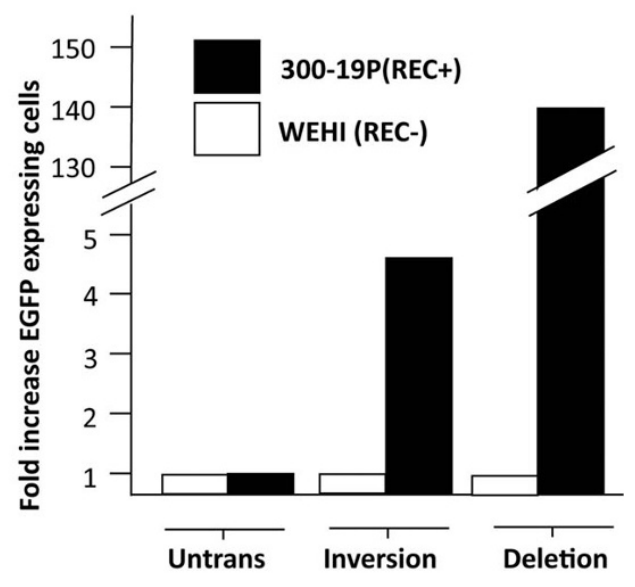

Figure 3 Expression of the recombined substrates. (a) Fluorescence microscopy of EGFP and DsRed expression. The four panels show expression of the DsRed (from unrearranged template) and rearranged EGFP gene products from inversion and deletion substrates in both 300$19 \mathrm{P}$ and WEHI231 cells. (b) Flow cytometric analysis of 4-week cultures of untransfected and transfected recombinant 300-19P (REC+) and WEHI231 (REC-) cell lines. The fold increase in number of EGFP-expressing cells was established by assigning an arbitrary value of 1 for untransfected cells.

recombination shown in Figure $2 \mathrm{~b}$ was not quantitative. However, these PCR results also suggest a lower level of recombination of the inversion construct compared to the deletion construct, a conclusion that has previously been reached using extrachromosomal substrates [3]. However, inversion events are a normal process in the assembly of antigen receptor loci and can occur over megabase-length regions of the chromosome [13].

The process of $\mathrm{V}(\mathrm{D}) \mathrm{J}$ recombination has evolved to generate a repertoire of antigen receptors. Large repertoires are generated by both combinatorial diversity and by the imprecise nature of the recombination process whereby the location of the double strand break is not fixed and nucleotides can be added or deleted from the free DNA end, generating extensive diversity in complementarity determining region (CDR) 3 of the antigen receptor [14-16]. Cloning and sequencing of the coding joints from both deletion and inversion substrates transfected into 300-19P cells revealed that a number of independent recombination events had occurred in the transfected cell population and that recombination of both constructs bore the typical hallmarks of V(D)J recombination, namely the introduction of diversity at the recombination junction via insertion and deletion of sequences (Table 1).

In the system described here, the use of the twin fluorescent protein system distinguishes individual cells that have recombined a $\mathrm{V}(\mathrm{D}) \mathrm{J}$ recombination substrate from those which carry the substrate in a configuration representing germline gene segments. The ability to assay the absence of recombination is important when testing the effects of mutations or local chromatin environment on the recombination process. Importantly, the twin fluorescent protein system allows recombination to be detected in situ in the absence of selection. Previously, assays of $\mathrm{V}(\mathrm{D}) \mathrm{J}$ activity have relied heavily upon extrachromosomal recombination substrates that are transiently transfected into cells before recovery and transformation into E. coli [3-6]. Chromatin structure is important in regulating $\mathrm{V}(\mathrm{D}) \mathrm{J}$ rearrangement $[17,18]$ and future studies will benefit from the use of substrates, such as those describe here, that integrate into the chromosome and allow simple assessment of germline or rearranged configuration.

Interestingly, the twin fluorescent protein system also indicates that $\mathrm{V}(\mathrm{D}) \mathrm{J}$ recombination could be used to control the expression of exogenous genes. Just $67 \mathrm{bp}$ of $c i s-$ acting sequences (RSS12 + RSS23) need to be provided to ensure correct expression of the exogenous gene (unpublished results). The ability to switch gene expression from one gene to another by harnessing endogenous recombinase activity may have practical applications in the $\mathrm{B}$ and $\mathrm{T}$ cell lineage analogous to the use of the Cre/ Lox system or other exogenous recombinases [19]. 
Table 1 Diversity at coding joint sequences

\begin{tabular}{|c|c|c|c|c|}
\hline RSS23 coding end sequence & Nucleotides lost & Nucleotides added & Nucleotides lost & RSS12 coding end sequence \\
\hline ATTACGCGC* & & & & ${ }^{*}$ GGTACCGTC \\
\hline \multicolumn{5}{|l|}{ Deletion substrate: } \\
\hline ATTACGCG & $-C$ & $+\mathrm{GC}$ & $-G$ & GTACCGTC \\
\hline ATTACG & $-C G C$ & & $-G G$ & TACCGTC \\
\hline ATTACGC & $-G C$ & & -GGTAC & CGTC \\
\hline ATTACGCG & $-C$ & $+\mathrm{GG}$ & -GGTA & CCGTC \\
\hline ATTACGCG & $-C$ & & $-G G$ & TACCGTC \\
\hline ATTACGCG & $-C$ & $+\mathrm{GCG}$ & -GGTA & CCGTC \\
\hline ATTACG & $-C G C$ & & $-G$ & GTACCGTC \\
\hline ATTACGCG & $-C$ & & $-G$ & GTACCGTC \\
\hline ATTACGCG & $-C$ & & $-G$ & GTACCGTC \\
\hline \multicolumn{5}{|l|}{ Inversion substrate: } \\
\hline ATTACGCGC & $-C$ & $+\mathrm{G}$ & -GGTA & CCGTC \\
\hline ATTACG & $-C G C$ & & $-G G$ & TACCGCG \\
\hline ATTACGCG & $-C$ & $+\mathrm{GC}$ & -GGT & ACCGTC \\
\hline ATTACGCG & $-C$ & & & GGTACCGTC \\
\hline ATTACGCG & $-C$ & $+\mathrm{GC}$ & -GGT & ACCGTC \\
\hline ATTACGCG & $-C$ & $+\mathrm{GC}$ & -GGT & ACCGTC \\
\hline ATTACG & $-C G C$ & & $-G G$ & TACCGTC \\
\hline ATTACGC & $-G C$ & & -GGTAC & CGTC \\
\hline ATTACGCG & $-C$ & $+\mathrm{GC}$ & $-G$ & GTACCGTC \\
\hline ATTACGCG & $-C$ & & $-G$ & GTACCGTC \\
\hline
\end{tabular}

DNA is cleaved at the boundary of the recombination signal sequence (RSS) motifs and the ends are joined. Joining involves the trimming of nucleotides and the addition of bases via terminal deoxynucleotide transferase (TdT), resulting in diversity at the coding joint.

*The sequences marked indicate the predicted sequence if recombination involved no nucleotide loss or addition. The actual sequences obtained for the deletion and inversion substrate are shown, with nucleotides loss and gained indicated.

\section{Methods}

\section{Construction of recombination substrates}

The inversion and deletion plasmids (shown in Figure 2) were constructed using pJMA2EGFP [20] and pDsRedN1 (Clontech-Takara Bio Europe, Saint-Germain-enLaye, France). Briefly, a double stranded oligonucleotide containing RSS12 was inserted upstream of DsRed, between the promoter and coding sequence. The EGFP sequence from pJMA2EGFP was then inserted downstream of DsRed, either on the same strand as DsRed (for the deletion construct) or on the opposite strand (for the inversion construct). An RSS23 motif was then added (using a double stranded oligonucleotide) either downstream of EGFP (for the inversion plasmid) or between DsRed and EGFP (for the deletion plasmid). The sequences of the RSS 12 and RSS 23 motifs are shown in Table 2. The precise details of the plasmid

Table 2 Recombination signal sequence (RSS) motifs

\begin{tabular}{ll}
\hline Signal & Sequence \\
\hline RSS12 & CACAGTGctacagactggaACAAAAACC \\
RSS23 & CACAGTGgtagtactccactgtctggctgtACAAAAACC \\
\hline
\end{tabular}

RSS motifs are comprised of a conserved heptamer and conserved nonamer (shown in upper case) separated by either 12 or 23 base pairs (bp) of intervening DNA (lower case). construction, including oligonucleotide sequences used, are available on request from the authors.

\section{Cell culture and transfection}

Murine B cell lineage cell lines (300-19P and WEHI231) were maintained in RPMI medium supplemented with $10 \%$ foetal calf serum and $50 \mu \mathrm{M} 2$-mercaptoethanol. The recombination substrates were transfected into preB (300-19P) and B (WEHI231) cell lines by electroporation. Approximately $20 \mu \mathrm{g}$ of linearised DNA were electroporated $(350 \mathrm{~V}, 700 \mu \mathrm{F})$ into $10^{7}$ cells in a volume of $0.8 \mathrm{ml}$ of medium. Cells were then incubated for $16 \mathrm{~h}$ in a total volume of $10 \mathrm{ml}$ of medium before the addition of $\mathrm{G} 418(2 \mathrm{mg} / \mathrm{ml})$ for selection.

\section{Nucleic acid isolation and PCR}

DNA and RNA were isolated from $10 \mathrm{ml}$ aliquots of culture using Genelute kits (Sigma-Aldrich Ltd, Poole, UK) as per the manufacturers' protocols. The optical density was measured at $260 \mathrm{~nm}$ and the concentration of the nucleic acid calculated; $500 \mathrm{ng}$ of genomic DNA was used as template in a PCR reaction. Recombination events were detected by PCR using appropriate primers (shown in Figure 1) for 35 cycles of $94^{\circ} \mathrm{C}(1 \mathrm{~min}), 58^{\circ} \mathrm{C}$ $(1 \mathrm{~min})$ and $72^{\circ} \mathrm{C}(1 \mathrm{~min})$ with a final extension step of 
10 min at $72^{\circ} \mathrm{C}$. PCR products encompassing the coding joint region of the recombined substrates were cloned into pcDNA3 and sequenced. For RT-PCR, reverse transcriptase (Invitrogen, Paisley, UK) was used to synthesise the first strand cDNA from $5 \mu \mathrm{g}$ of total RNA using random primers (alongside negative controls lacking enzyme). The first strand synthesis reaction was then diluted fivefold and used as a template for PCR reactions to detect mRNA transcribed from rearranged DNA substrates (primer sequences available on request from the authors).

For RT-PCR of mouse genes, the following primer sequences were used; RAG1F, CCCGATGAAATTCAACACCC; RAG1B, CTTGACTTCCCATCAGCATGG; RAG2F, CCTGTCCTACTGGAGTCTTTC; RAG2B, GGCCGTATCTGGGTTCAGGG; $\beta$-actin F, TGCGTGACATCAAAGAGAAG; $\beta$-actin B, GATGCCACAGGATTCCATA. Reactions with these primers were performed as described for recombination assays.

\section{Flow cytometry}

Cells transfected with deletion or inversion substrates were analysed for the expression of the EGFP protein (resulting from recombination of the substrate) using the FL1 channel of a FACS Calibur flow cytometer (BD Biosciences, Oxford, UK). The mean fluorescence intensity of EGFP expression was compared between transfected and non-transfected cells and the data expressed as a fold increase in fluorescence with non-transfected cells assigned an arbitrary value of 1 .

\footnotetext{
Acknowledgements

This work was supported by a Wellcome Trust Showcase Award to GPC. We would like to thank J Askham for providing us with the EGFP vector.
}

\section{Authors' contributions}

GPC designed the overall strategy, GPC and GBS designed the constructs, GBS performed the experiments, EADW assisted with flow cytometry and GPC and GBS wrote the paper.

\section{Competing interests}

The authors declare that they have no competing interests.

Received: 24 March 2009 Accepted: 1 March 2010

Published: 1 March 2010

\section{References}

1. Gellert $M: V(D) J$ recombination: RAG proteins, repair factors, and regulation. Annu Rev Biochem 2002, 71:101-132.

2. Hesse JE, Lieber MR, Mizuuchi K, Gellert M: V(D)J recombination: a functional definition of the joining signals. Genes Dev 1989, 3:1053-1061.

3. Hesse JE, Lieber MR, Gellert M, Mizuuchi K: Extrachromosomal DNA substrates in pre-B cells undergo inversion or deletion at immunoglobulin V-(D)-J joining signals. Cell 1987, 49:775-783.

4. Gauss GH, Lieber MR: Unequal signal and coding joint formation in human V(D)J recombination. Mol Cell Biol 1993, 13:3900-3906.

5. Shockett P, Difilippantonio M, Hellman N, Schatz DG: A modified tetracycline-regulated system provides autoregulatory, inducible gene expression in cultured cells and transgenic mice. Proc Natl Acad Sci USA 1995, 92:6522-6526.
6. Dobbeling U: The influence of IL-7 V(D)J recombination. Immunology 1996, 89:569-572.

7. Blackwell TK, Alt FW: Site-specific recombination between immunoglobulin $\mathrm{D}$ and $\mathrm{JH}$ segments that were introduced into the genome of a murine pre-B cell line. Cell 1984, 37:105-112.

8. Blackwell TK, Moore MW, Yancopoulos GD, Suh H, Lutzker S, Selsing E, Alt FW: Recombination between immunoglobulin variable region gene segments is enhanced by transcription. Nature 1986, 324:585-589.

9. Engler P, Storb U: High-frequency deletional rearrangement of immunoglobulin kappa gene segments introduced into a pre-B-cell line. Proc Natl Acad Sci USA 1987, 84:4949-4953.

10. Borghesi L, Hsu LY, Miller JP, Anderson M, Herzenberg L, Schlissel MS, Allman D, Gerstein RM: B lineage-specific regulation of $V(D) J$ recombinase activity is established in common lymphoid progenitors. J Exp Med 2004, 199:491-502.

11. Zheng X, Schwarz K: Making V(D)J rearrangement visible: quantification of recombination efficiency in real time at the single cell level. $J$ Immunol Methods 2006, 315:133-143.

12. Bredemeyer AL, Sharma GG, Huang CY, Helmink BA, Walker LM, Khor KC, Nuskey B, Sullivan KE, Pandita TK, Bassing CH, Sleckman BP: ATM stabilizes DNA double-strand-break complexes during $\mathrm{V}(\mathrm{D}) \mathrm{J}$ recombination. Nature 2006, 442:466-470.

13. Weichhold GM, Klobeck HG, Ohnheiser R, Combriato G, Zachau HG: Megabase inversions in the human genome as physiological events. Nature 1990, 347:90-92.

14. Alt FW, Baltimore D: Joining of immunoglobulin heavy chain gene segments: implications from a chromosome with evidence of three D-JH fusions. Proc Natl Acad Sci USA 1982, 79:4118-4122.

15. Tonegawa S: Somatic generation of antibody diversity. Nature 1983, 302:575-581

16. Lieber MR, Hesse JE, Mizuuchi K, Gellert M: Lymphoid V(D)J recombination: nucleotide insertion at signal joints as well as coding joints. Proc Nat/ Acad Sci USA 1988, 85:8588-8592.

17. McBlane F, Boyes J: Stimulation of V(D)J recombination by histone acetylation. Curr Biol 2000, 10:483-486.

18. Cobb RM, Oestreich KJ, Osipovich OA, Oltz EM: Accessibility control of V (D)J recombination. Adv Immunol 2006, 91:45-109.

19. Tronche F, Casanova E, Turiault M, Sahly I, Kellendonk C: When reverse genetics meets physiology: the use of site-specific recombinases in mice. FEBS Lett 2002, 529:116-121.

20. Askham JM, Moncur P, Markham AF, Morrison EE: Regulation and function of the interaction between the APC tumour suppressor protein and EB1. Oncogene 2000, 19:1950-1958.

doi:10.1186/1759-8753-1-9

Cite this article as: Scott et al.: Detecting variable (V), diversity (D) and joining (J) gene segment recombination using a two-colour fluorescence system. Mobile DNA 2010 1:9.

\section{Submit your next manuscript to BioMed Central and take full advantage of:}

- Convenient online submission
- Thorough peer review
- No space constraints or color figure charges
- Immediate publication on acceptance
- Inclusion in PubMed, CAS, Scopus and Google Scholar
- Research which is freely available for redistribution

Submit your manuscript at www.biomedcentral.com/submit
Ciomed Central 